The Injury Prevention Research Unit funded by the Accident Rehabilitation and Compensation Insurance Corporation, and the Health Research Council (HRC) of New Zealand. Dr Langley is a HRC principal research fellow. The authors wish to thank John Eastwood, David Chalmers, and Lesley Day for thank John Eastwood, David Chalmers, and Lesley Day
their useful comments on an earlier version of this paper.

1 Schelp L. Epidemiology as a basis for evaluation of a community intervention programme on accidents. Sund byberg, Sweden: Karolinska Institute, Department of Social Medicine, 1987

2 Svanström L, Ekman R, Schelp L, Lindström A. The Lidkoping Accident Prevention Programme - a com munity approach to preventing childhood injuries in Sweden. Injury Prevention 1995; 1: 169-72.

3 Ozanne-Smith J, Sherrard J, Brumen IA, Vulcan P. Community based injury prevention evaluation report: Shire of Bulla Safe Living Program. The first three years 1991-1993. Melbourne: Monash University Accident Research Centre, 1994.

\section{Commentary: statistical perspectives on the Lidköping papers}

We have examined the critique by Langley and Alsop and the original Lidköping paper and re-entered the data in order to replicate both sets of analyses. As we see it, the authors of the original paper (Svanström et al) do indeed lose statistical power by breaking the data down too far and performing two separate analyses (one for boys, one for girls). For each of the two genders, they find the difference in the trends over time between the intervention and neighbouring area to be non-significant $(-0.3$ $-0.2=-0.5 /$ year for girls, and -0.4 $-0 \cdot 1=-0.5 /$ year for boys). The $\mathrm{p}$ values are thus 0.33 and $0 \cdot 25$. Instead of reporting these $p$ values, they use words like 'strengthen the impression' and 'support the proposition'.

Before commenting on the method suggested by Langley and Alsop, we suggest two ways in which the original authors could have made more efficient use of their data. The first of these is to aggregate the genders and calculate one rate per year for the two genders combined. When we do this (either by a 'rougher' straight average of the two rates, or in a fancier way by adding numerators and adding denominators), we get somewhat stronger evidence of a difference in the two slopes. One way to test for a difference in slopes is to include an year $X$ area term in the model in addition to area and year - just as Langley and Alsop did. The single $p$ value is $0 \cdot 18$, corresponding to a 'difference in slopes' of $-0.47 /$ year. In the critique from New Zealand the authors give a $p$ value of 0.041 for this interaction in the Poisson model. They fail, however, to tell the reader in which direction this 'difference of slopes' goes. We hope that the journal will set a firm tone by asking for magnitude and direction first, confidence interval $(\mathrm{CI})$ second, and $\mathrm{p}$ values last (if at all).

The second approach, still staying with rates, is to treat the rates in boys and girls as one data set, but to account for (that is, take out), the variation in the rates between the two genders by including gender as a term in the regression. In PROC GLM in SAS for example, one can model the rates, rate $=$ gender year area year $x$ area.

If one does this with the 36 data points in the first four columns of the table in the paper, and if one codes area as 1 if intervention and 0 otherwise, and gender as 1 if boys and 0 if girls, and if we center the years by subtracting 1987 so that 1983 is -4 and 1991 is +4 , we get the following fit (SEs in parenthesis):

$$
\begin{aligned}
& \text { rate }=8.5 \\
& +4.3(0.78) \text { gender } \\
& +0.12(0.21) \text { year } \\
& +1.69(0.78) \text { area } \\
& -0.48(0.30) \text { year } \times \text { area. }
\end{aligned}
$$

Notice that the coefficient for area is +1.69 , indicating that, in general, rates are higher in the intervention area. The key is the -0.48 (very close to the average of the two gender specific differences in slopes in the authors' analysis. The $t$ statistic for this is -1.6 so the $p$ value (from a $t$ with $31 \mathrm{df}$ ) is $0 \cdot 12$.

Before dealing in depth with the submission from New Zealand, two preliminary comments. First, we believe that - from a purely data analytic viewpoint - they are wise to treat the 36 observations (intervention $v$ neighbouring area) as one data set rather than two (one for each gender). Second, however, we would have liked to see coefficients, not just $p$ values.

Now, to the main issue, which is the use of a Poisson model. There is a paradox here in that the authors find evidence of some extraPoisson variation when the genders are considered separately, yet mysteriously these problems 'go away' when all 36 data points are considered together. The issue of whether the Poisson distribution is - in principle, and in this data set - the 'correct' model to use is an important one. The Poisson model appears to 'bring out the signal' better than the analysis that treats the variations around the lines as Gaussian. If we were dealing with counts that were unlikely to be influenced by factors such as weather, 'local' short term interventions, clustering because of injuries to multiple persons from the same source/cause (for example, an incident with a school bus), or any other such perturbations, then the Poisson distribution would make sense. But a priori we would have expected extra-Poisson variation and suspect that the reason the authors don't 'find' it is that it is buried in the large number of degrees of freedom.

Indeed, when we look more closely, the ratio of deviance/df of 1.79 tells us that the deviance is around 55, which is beyond the $p=0.01$ point of the $\chi^{2}$ distribution with $31 \mathrm{df}$. So, in fact, the Poisson model does not fit that well: there is more variation than the Poisson model would predict. Thus, the standard errors obtained for the coefficients for the model in table 2 are too small, and the $\mathrm{p}$ values too extreme. Indeed, it appears that the more naive model in the original paper, which considers the variance around the line to have a magnitude independent of (bigger than) the mean, is more appropriate in this case. In general, it makes more sense to use a model that allows the variation to be estimated from the data rather than from an assumption that is not fulfilled by the data. The model used in our analysis above is the same as the one used by the original authors, except that we use all the data in one analysis.

In spite of using all the data at once, and 
using the model which fits the data (rather than using standard errors calculated from a model that doesn't fit the data), we too are unable to show that the difference of slopes is significant at the 'magic' 0.05 level. Thus, even though we agree with translating betas (and differences of betas) into numbers of injuries, we wonder if these numbers shouldn't be taken with a grain of salt. After all, from our conclusion that the difference in betas isn't statistically significant at the 0.05 level, we can project that the $95 \% \mathrm{CI}$ for the number of injuries prevented will include zero.

The paper from New Zealand argues that a Gaussian distribution may not be appropriate, whereas a Poisson one would. In fact, the two are not mutually exclusive. If one had a Poisson distribution with a mean of 33 or 50 events per year (or $>80$ if we combine the two genders), then the distribution is also, for all intents and purposes, Gaussian. The main point of a Poisson distribution is that it is a one parameter distribution in which the variance equals the mean - something that appears not to be the case with the data in this application.

A cautionary note: if we fuss too much about the distinctions between these two forms of error variation, or between linear and log linear regression, or indeed other issues in modelling, we run the risk of concentrating too much on the 'small picture'. A much bigger uncertainty in the inferences we can draw from this study stems from the ecologic nature of the data and the fact that it is an 'unrandomized' study of $\mathrm{n}=2$ units (areas), each of which is subject to many other influences beyond those allowed for, or allowable for, in the analysis. So we should keep these arguments about models and $p$ values in perspective: the results in the county as a whole are another sobering reminder of what else might be going on that our 'model' cannot account for.

In summary, the New Zealand authors appear to squeeze too much 'statistical significance' from the data by using a model that artificially makes the standard errors too small and the $p$ values too extreme. The original authors could have used the data to their full advantage. However, if they had tried harder (as we did), by analyzing all of the 36 data points at once, the result would have remained 'NS'. But even if they had found $\mathrm{p}=0.04$ rather than the 0.12 or some other such value, we would still need to be cautious in our interpretation.

JAMES HANLEY SUNGSUB CHOI Department of Epidemiology and Biostatistics, McGill University, Purvis Hall, 1020 Pine Avenue Montreal, Quebec $H 3 A 1 A 2$ Canada

\section{Believe it or not but this really happened ...}

A CCSN BBS correspondent writes: 'Because I am an in-line instructor who is very concerned with the issue of safety I tend to find myself in these situations. I was skating at an indoor rink when I saw this father readying his young daughter for skating. She was wearing all of the protective gear, a rarity for most indoor establishments. When I noticed them Dad was trying to put a helmet on the little girl's head. Part of the difficulty he was having stemmed from the fact that the helmet was backwards.

You've really got to learn the correct way to wear a helmet, and because it's not apparent to everybody, I always try to be helpful. I skated over to the father and told him that his daughters' helmet was on backwards. He told me that it was 'okay', I thought maybe he didn't hear me as the music sometimes gets quite loud. I tried again. I explained that the back of the helmet is the side with the most styrofoam, to cushion the base of the skull in a backwards fall. Dad thanked me very politely, and explained that it didn't matter, as his daughter was not capable of skating fast enough for it to be an issue. Well how do you argue with logic like that? Apparently he thought she'd be travelling fast enough to require a helmet, but not quite fast enough to require she wear it correctly.

Well Dad was right about one thing - the little girl didn't skate very fast - [and] the other kids were darting about her like 'bats out of Hell'.

Every now and then one of those kids would get a little too close and that little girl would be on the ground. It didn't take long for the helmet to slip off her head and dangle about her neck like the Red Baron's scarf. I watched Dad race out and reaffix the still backwards helmet several times. I tried to explain to him that helmets are designed to fit the head a specific way, and that maybe it would stay on better if he turned it around. He felt her hair was making it slip off and again he would be more concerned about it being on correctly if she could skate faster. (There was that wacky logic again.)

After about the ninth attempt to reaffix the helmet Dad decided to try turning the helmet the other way. After she skated the two laps without incident he announced that maybe it did fit better when it was on correctly' (CCSN BBS). 